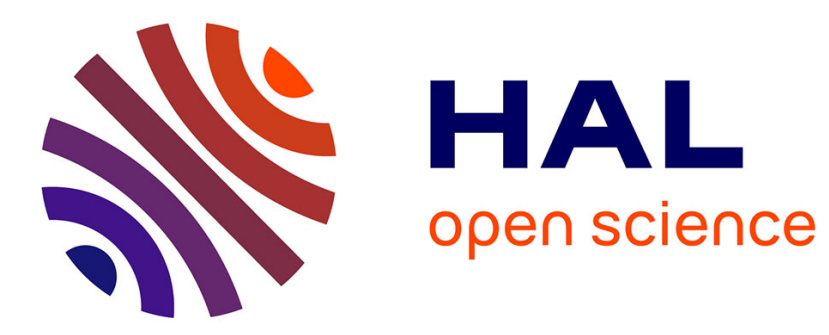

\title{
Removing quasi-periodic noise in strain maps by filtering in the Fourier domain
}

Michel Grediac, Frédéric Sur, Benoît Blaysat

\section{To cite this version:}

Michel Grediac, Frédéric Sur, Benoît Blaysat. Removing quasi-periodic noise in strain maps by filtering in the Fourier domain. Experimental Techniques, 2016, 40 (3), pp.959-971. 10.1007/s40799-016-01002. hal-01163838

\section{HAL Id: hal-01163838 \\ https://hal.inria.fr/hal-01163838}

Submitted on 16 Jun 2015

HAL is a multi-disciplinary open access archive for the deposit and dissemination of scientific research documents, whether they are published or not. The documents may come from teaching and research institutions in France or abroad, or from public or private research centers.
L'archive ouverte pluridisciplinaire $\mathbf{H A L}$, est destinée au dépôt et à la diffusion de documents scientifiques de niveau recherche, publiés ou non, émanant des établissements d'enseignement et de recherche français ou étrangers, des laboratoires publics ou privés. 


\title{
Removing quasi-periodic noise in strain maps by filtering in the Fourier domain
}

\author{
M. Grédiac ${ }^{1 \dagger}$, F. Sur ${ }^{2}$, B. Blaysat ${ }^{1}$ \\ ${ }^{1}$ Clermont Université, Université Blaise Pascal, Institut Pascal, UMR CNRS 6602 \\ BP 10448, 63000 Clermont-Ferrand, France \\ ${ }^{2}$ Laboratoire Lorrain de Recherche en Informatique et ses Applications, UMR CNRS 7503 \\ Université de Lorraine, CNRS, INRIA projet Magrit, Campus Scientifique, BP 239, 54506 \\ Vandoeuvre-lès-Nancy Cedex, FRANCE \\ † SEM member, corresponding author, tel: +3347328 80 77, fax: +33473288027, \\ michel.grediac@univ-bpclermont.fr
}

\begin{abstract}
Quasi-periodic noise due to various reasons often corrupts strain maps obtained with full-field measuring systems. The aim of this didactic paper is to show how to remove this noise by changing some Fourier coefficients involved in the 2D Fourier transform of these strain maps. The basics of the 2D Fourier transform of images, which is a common tool in image processing but which is only scarcely employed in the experimental mechanics community, are first briefly recalled. Several procedures employed for removing undesirable frequencies in strain maps are then discussed. Three different examples illustrate the benefit of this approach.
\end{abstract}

Keywords: Fourier transform, grid method, image processing, strain measurement, strain map restoration

This document is a preprint version of an article published in Experimental Techniques (Wiley): DOI: 10.1111 /ext.12162

http://onlinelibrary.wiley.com/doi/10.1111/ext.12162/abstract 


\section{Introduction}

Full-field measurement techniques rapidly spread in the experimental solid mechanics community, so measuring reliable displacement and strain fields on the surface of deformed specimens becomes an increasingly relevant issue. Various techniques are available such as moiré, moiré interferometry, speckle interferometry, the grid method and digital image correlation [1]. These techniques rely either on the use of lasers and the interference phenomenon, or on white light and geometric optics. This paper mainly concerns methods based on fringe or regular marking processing. In these cases, the sought quantities (displacement and strain components) are encoded in the phase (for the displacement) or in the spatial derivatives of the phase (for the strain), so retrieving them requires the use of procedures based on Fourier analysis [2]. Strain maps obtained with these techniques, especially with the grid method, are sometimes impaired by parasitic fringes, which can be considered as a quasi-periodic noise [3]. They may be due to grid defects or to sampling issues such as aliasing when grid images are shot by the camera. It is finally worth mentioning that when digital image correlation is used, interpolation error may also cause such periodic noise called matching bias, which appears in the strain maps $[4,5]$.

These unwanted side effects lead to parasitic fringes in strain maps. These fringes feature various forms in terms of shape, frequency, intensity and orientation. Regardless of the physical cause, the practical question for the user is to know how to remove this quasi-periodic noise when it corrupts strain maps. Directly tackling the cause to remove this effect is the most efficient route. However, when these causes remain obscure or unavoidable, another possibility is to work directly on the strain maps to remove them using suitable image processing tools. When the frequency of the noise is high and the actual strain gradient small, locally smoothing with polynomials [6] or with a low-pass spatial filter is possible. However if the actual features in the strain maps exhibit a "characteristic length" of the same order of magnitude as the period of the fringes (or obviously lower), smoothing and spatial filtering have a strong impact on the actual strain values: details are smoothed (or even lost). The amplitude of the displayed value on the final map becomes also lower than the actual one. An alternative in this case is to employ a filter in the frequency domain by taking the Fourier transform of the strain map and judiciously correcting the spectrum in the frequency domain.

In this context, the objective of this paper is to show how to proceed in practice to perform this filtering, in a context for which handling Fourier transforms of images is not really familiar in the experimental mechanics community. In addition, a huge literature is available in the image processing community on the topic of white noise removal (see, e.g., the review papers $[7,8]$ ), and the removal of a subclass of stationary noises (convolved white noises) has been recently addressed in [9]. However, the case of structured, quasi-periodic noises, which cannot be modeled as stationary processes, is the subject of a limited literature (reminded in Section 3.1) and leads to less sophisticated algorithms [10].

The paper is organized as follows. Some basics on the the 2D-discrete Fourier transform are recalled. Various procedures which enable removal of quasi-periodic noise in strain maps are then presented. Three examples illustrate the merits and limits of this approach. Note finally that displacement maps may potentially be filtered with the same procedure, but the problem of parasitic fringes arises in practice in strain maps, not in displacement maps, so only strain maps are considered herein.

\section{A brief reminder about the discrete Fourier transform}

For the sake of completeness, this section is a reminder about the Fourier analysis of a 2D discrete signal and its basic properties. The case of periodic wave-like patterns, which is the topic of the paper, is also discussed. 


\subsection{Notations and basic properties}

Let be a $2 \mathrm{D}$ signal $s$ made of $M \times N$ samples, typically a $M \times N$ gray level image, or here a discretized strain map obtained with a full-field measurement system, defined such as:

$$
\begin{aligned}
\{1,2, \ldots, M\} \times\{1,2, \ldots, N\} & \rightarrow \mathbb{R} \\
(x, y) & \mapsto s(x, y)
\end{aligned}
$$

The discrete Fourier transform of $s$ is defined as the function $\widehat{s}$ :

$$
\begin{aligned}
\mathbb{N}^{2} & \rightarrow \mathbb{R} \\
(u, v) & \mapsto \widehat{s}(u, v)=\sum_{x=0}^{M-1} \sum_{y=0}^{N-1} s(x, y) e^{-2 \pi i(x u / M+y v / N)}
\end{aligned}
$$

Note that the whole problem is written in terms of pixels numbering, meaning that $(x, y)$ (given in pixel) and $(u, v)$ (given in pixel $^{-1}$ ) have integer values and have no physical unity, as well as $M$ and $N$.

The inverse Fourier transform writes as follows

$$
s(x, y)=\frac{1}{M N} \sum_{u=0}^{M-1} \sum_{v=0}^{N-1} \widehat{s}(u, v) e^{2 \pi i(x u / M+y v / N)}
$$

By definition, both $s$ and $\hat{s}$ have the same dimensions. It is also important to note that adding any integer multiple of $M$ to $u$ or of $N$ to $v$ lets the value of $\widehat{s}$ unchanged. The discrete Fourier transform $\widehat{s}$ can thus be seen as a $(M, N)$-periodic function. In an equivalent way, the inverse Fourier transform formula implicitly makes $s$ D periodic. When $(u, v) \in\left[-\left(M / 2+\alpha_{M}\right), M / 2+\right.$ $\left.\beta_{M}\right] \times\left[-\left(N / 2+\alpha_{N}\right), N / 2+\beta_{N}\right]$, where $\alpha_{i}=\frac{-1+(-1)^{i}}{4}$ and $\beta_{i}=-\frac{3+(-1)^{i}}{4}, i=M, N,\left(\alpha_{i}\right.$ and $\beta_{i}$ are added to take into account the parity of $N$ and $\left.M\right)|\widehat{s}(u, v)|$ is the amplitude of a periodic component of frequencies $|u| / M$ and $|v| / N$ of $s$ in each direction. The norms $|\widehat{s}(u, v)|$ for $u$ (resp. $v)$ spanning an interval of length $M($ resp. $N)$ give an image called the amplitude spectrum of $s$. Similarly, $|\widehat{s}|^{2}$ is called the power spectrum of $s$.

If the 2D signal $s$ is real, then the complex conjugate $\bar{s}$ of $\widehat{s}$ satisfies

$$
\overline{\widehat{s}}(u, v)=\sum_{x=0}^{M-1} \sum_{y=0}^{N-1} s(x, y) e^{2 \pi i(x u / M+y v / N)}=\widehat{s}(-u,-v)
$$

Consequently, the (amplitude or power) spectrum is symmetric with respect to $(\xi, \eta)=(0,0)$. Since the spectrum is actually a periodic function, the origin is quite arbitrary when visualizing the results. It is generally chosen to be at the center of the visual representation. This leads to a symmetry with respect to the center of the image.

Let us also precise the role of a rotation $s_{\theta}$ of angle $\theta$ of the $2 \mathrm{D}$ signal $s$. A simple calculation shows that the Fourier transform of a rotated continuous 2D signal is the Fourier transform of the original signal, rotated by the same angle. Apart from (potentially noticeable) discretization effects, this means that the spectrum of a rotated image is the spectrum of the original image rotated by the same angle.

\subsection{Fourier transform of a sine wave}

A discrete sine or cosine $2 \mathrm{D}$ function of frequency $f$ (between $-1 / 2$ and $1 / 2$ ) and phase difference $\phi$ is such that

$$
s(x, y)=A \cos (2 \pi f x+\phi)=\frac{A}{2}\left(e^{i(2 \pi f x+\phi)}+e^{-i(2 \pi f x+\phi)}\right)
$$

with $x$ and $y$ taking respectively $M$ and $N$ integer values. From the remark on rotations in the previous paragraph, we can consider a wave along the $x$-direction without loss of generality. The Fourier transform of $s$ writes as follows

$$
\widehat{s}(u, v)=\frac{A}{2} \sum_{x=0}^{M-1}\left(e^{2 \pi i x(f-u / M)+i \phi}+e^{-2 \pi i x(f+u / M)-i \phi}\right) N \delta_{0}(v)
$$


where $\delta_{0}(v)=1$ if and only if $v=0$, since $\sum_{y=0}^{N-1} e^{-2 \pi i y v / N}=0$ if $v \neq 0$ and $=N$ if $v=0$.

Moreover, if $f=u / M$, then $\sum_{x=0}^{M-1} e^{2 \pi i x(f-u / M)}=M$ and if $f \neq u / M$, then $\sum_{x=0}^{M-1} e^{2 \pi i x(f-u / M)}=$ $\left(1-e^{2 \pi i(f M-u)}\right) /\left(1-e^{2 \pi i(f-u / M)}\right)$. Hence, two cases are possible

- Either $f$ is a multiple of $1 / M(f=k / M)$. In this case $e^{2 \pi i(f M-u)}=1$, and we can conclude that

$$
\widehat{s}(u, v)=M N \frac{A}{2}\left(e^{i \phi} \delta_{k}(u)+e^{-i \phi} \delta_{-k}(u)\right) \delta_{0}(v)
$$

where $\delta_{ \pm k}(u)=1$ if and only if $u= \pm k$ (modulo $M$ ) and $\delta_{ \pm k}(u)=0$ otherwise.

The spectrum of $s$ shows therefore two symmetric peaks (corresponding to the two non-null Fourier coefficients) at $( \pm k, 0)= \pm(f M, 0)$, such that the frequency of the wave is $f=k / M$.

- Or $f$ is not a multiple of $1 / M$. In this case,

$$
\begin{aligned}
\widehat{s}(u, v)= & \frac{A}{2}\left(e^{i \phi} \frac{1-e^{2 \pi i(f M-u)}}{1-e^{2 \pi i(f-u / M)}}+e^{-i \phi} \frac{1-e^{-2 \pi i(f M+u)}}{1-e^{-2 \pi i(f+u / M)}}\right) N \delta_{0}(v) \\
= & \frac{A}{2}\left(e^{i(\phi+\pi(f M-u-f+u / M))} \frac{\sin (\pi(f M-u))}{\sin (\pi(f-u / M))}\right. \\
& \left.+e^{-i(\phi+\pi(f M+u-f-u / M))} \frac{\sin (\pi(f M+u))}{\sin (\pi(f+u / M))}\right) N \delta_{0}(v)
\end{aligned}
$$

Since $\sin (\pi x) / \sin (\pi x / M)$ (defined for $x$ in $[-M / 2, M / 2-1]$ ) is an oscillating function attaining its maximum value $M$ for $x=0$, we can conclude that the spectrum of $s$ (with its origin at $(0,0))$ has two symmetric peaks around $( \pm f M, 0)$. These peaks are quite sharp but are not localized at a single coefficient as in the "ideal" case above.

Generally speaking, if two more or less broad peaks can be seen in the spectrum, the direction they define is likely to correspond to that of the propagation of parasitic fringes.

\subsection{Illustrative example}

We consider now the amplitude spectrum and phase distribution of any strain map as an illustrative example. The specimen shown in Figure 1-a is considered. It is subjected here to a vertical compression. This leads to the vertical strain map $\epsilon_{y y}$ which is measured with the grid method. It defines here the 2D signal $s$. A typical example of such a strain map is depicted in Figure 1-b [11]. The shape of the aggregates is clearly recognizable in this map. This is due to the fact that the strain is mainly concentrated in the binder, the stiffness of the latter being much lower than that of the aggregates. The amplitude spectrum of Figure 1-a, which corresponds to the distribution of $|\widehat{s}(u, v)|$, is shown in Figure 1-c, where the origin is at the center of the figure. The logarithmic scale is employed here to account for the significant difference in norm which is generally observed between the norms of the coefficients of low and high frequencies. This spectrum corresponds to the "signature" of the strain map in the frequency domain. With the convention mentioned above, the coefficients of the lowest frequencies are located near the center of the spectrum. The frequency increases when moving away from the center. The (unique) coefficient located at the center is the spatial average of the signal, here the mean strain. It can be easily checked here that the coefficients of the lowest frequencies exhibit the highest norm, which is a common feature of natural images [12]. The distribution of the argument is now shown in Figure 1-d. It is antisymmetric, contrary to the spectrum which is symmetric. This illustrates the result given in Equation 2.4. Many other properties of the Fourier transform of images can be found in [13]. The objective is now to explain how to rely on the discrete Fourier transform to get rid of parasitic periodic noise in strain maps. The native Matlab functions which were employed to perform this transform are listed in the Annex. 


\section{$3 \quad$ Filtering parasitic fringes or periodic noise in strain maps}

\subsection{Principle}

As explained in the introduction, strain maps obtained by processing fringes and grids (or, as a general remark, any repetitive and quasi-periodic patterns) by windowed Fourier transform sometimes feature some parasitic and annoying fringes, which can be considered as a periodic or quasi-periodic noise that corrupts thus these maps. The same phenomenon may also potentially occur in strain maps obtained by digital image correlation. The objective here is to show how to remove them from a strain map by relying on the Fourier transform recalled above. In certain cases, such a procedure is more efficient than local smoothing because actual details are expected not to be impacted, contrary to smoothing which affects both undesirable noise and actual details at the same time. If we consider that a map $s_{0}$ is corrupted by a periodic noise denoted here $w$, the resulting map $s$ is merely $s=s_{0}+w$. The discrete Fourier transform being linear, we have $\hat{s}=\widehat{s_{0}+w}=\hat{s_{0}}+\hat{w}$. Removing $w$ from $s$ is then theoretically very simple since it consists of:

1. calculating the discrete Fourier transform $\hat{s}$ of the initial map $s=s_{0}+w$ corrupted by $w$;

2. representing the Fourier amplitude spectrum in the frequency domain;

3. detecting the coefficients corresponding to the Fourier transform of the undesirable noise $\hat{w}$;

4. erasing these peaks;

5. calculating the inverse Fourier transform of the modified spectrum to get the filtered map.

The undesirable periodic noise is eventually expected to vanish from the maps, or at least to be strongly reduced. It is clear that step 4 above also affects some actual values for the Fourier coefficients of the actual map $s_{0}$. Consequently, depending on the procedure employed to erase the peaks, different results will be obtained for the filtered map. Previous papers have already described similar filtering procedures, for instance applied directly on pictures [3], on satellite images [14] or on maps corrupted by ultrasound artifacts in medical imaging [15]. In these cases however, the frequency of the periodic noise is generally higher and the corresponding peaks in the frequency domain are more isolated. The objective here is to examine how to employ the procedure described above on strain maps obtained with a contactless measuring technique, and to discuss the obtained results.

\subsection{Procedures for removing the peaks}

Parasitic fringes observed in strain maps are generally less regular than those appearing in other fields discussed above $[3,14,15]$. This leads steps 3 and 4 to be potentially more critical since the peaks to be erased are less isolated in the Fourier transform of strain maps. Concerning Step 3 above, this means that the automatic detection of the peaks described in some papers $([3,10]$ for instance) does not work well, while finding roughly "by hand" the zone of the spectrum affected by the periodic noise is quite easy: the period (and thus the frequency) of the fringes can indeed be estimated on the strain map, and since the direction of the peaks in the spectrum is the same as the direction of the waves corresponding to the noise (see 2.1 above), one can easily find the zone affected in the spectrum, as well as the zones located further away from the center potentially affected by the harmonics.

Noise considered here is a quasi-periodic function, but it is generally not rigorously a pure sine in practice. The amplitude spectrum therefore does not show two well isolated peaks (as recalled above, a given frequency is represented by a set of two peaks in the frequency domain, these peaks being symmetric with respect to the center of the spectrum), but rather two clusters of peaks surrounding their apices. Step 4 above can therefore be achieved by merely reducing, even zeroing the corresponding coefficient in the frequency domain. A point which can become critical is that the information on the actual strain distribution can be mixed with that concerning the parasitic fringes to be removed, thus the route followed to reduce the amplitude of the peaks may also affect and disturb the actual information. Several routes have therefore been tested here to select the peaks which must be corrected, and to choose a relevant value instead. They are either already 
available in the literature or merely based on common sense. Each of these procedures can be easily programmed using suitable native Matlab functions. Assuming a zone to be corrected in the spectrum has been chosen a priori, these procedures are as follows:

1. Procedure 1: replacing all the coefficients by zero in a zone (typically a rectangle) containing the peaks to be erased. This can be considered as a notch filter.

2. Procedure 2: replacing all these coefficients by the mean value (Procedure 2a) of the coefficients located along the one-pixel width strip surrounding this zone. The idea behind this correction is to account for the neighborhood of the zone to replace the coefficients within the zone by a relevant value which respects the global trend in the evolution of the coefficients around the zone. A variant can be to consider the median instead of the mean value, as suggested in [16] (Procedure 2b).

3. Procedure 3: replacing by the mean value (Procedure 3a) or by the median (Procedure 3b) only the coefficients whose norm is greater than a certain threshold value, and keep the others unchanged.

4. Procedure 4: replacing all the coefficients located inside the zone by a bilinear interpolation of the coefficients located along the strip of pixels surrounding this zone. As in Procedure 2, the idea is to account for the neighborhood of the zone to make the correction.

5. Procedure 5: applying a windowed Gaussian notch filter, as suggested in [3]. This can be made in practice by multiplying element-wise (Hadamard product) these coefficients by a value lower than one which is built upon a Gaussian function:

$$
\widehat{g}(u, v)=1-e^{-\left(\frac{u^{\prime 2}}{\sigma_{u}^{2}}+\frac{v^{\prime 2}}{\sigma_{v}^{2}}\right)}
$$

with

$$
\left\{\begin{array}{l}
u^{\prime}=\left(u-u_{p}\right) \cos (\theta)+\left(v-v_{p}\right) \sin (\theta) \\
v^{\prime}=-\left(u-u_{p}\right) \sin (\theta)+\left(v-v_{p}\right) \cos (\theta)
\end{array}\right.
$$

where $\left(u_{p}, v_{p}\right)$ represent the coordinates of the center of the cluster of peaks to be corrected, $\sigma_{u}$ and $\sigma_{v}$ the two standard deviations of the Gaussian, and $\theta$ an angle driving the orientation of the filter. The size of the zone affected by the correction may be limited to a rectangle or an ellipse, whose dimensions can be defined by a certain number of times $\sigma_{u}$ and $\sigma_{v}$. The idea here is to less abruptly correct the spectrum than in the preceding procedures. Indeed $\theta, \sigma_{u}$ and $\sigma_{v}$ can be adjusted in such a way that the filter is judiciously adapted to the shape of the cluster of peaks, using for instance a trial-and-error procedure. In addition, a smooth filter such as the one given by Equation 3.1 is less prone to ringing (Gibbs effect) than the filter of Procedure 1. Note finally that the zone symmetric with respect to the center in the spectrum must also be corrected;

Three examples are now briefly given to illustrate the effect of these procedures on strain maps.

\section{Examples}

\subsection{Example 1}

This example is a strain map obtained with the grid method, during a translation test carried out for characterizing sensor noise propagation of a camera, as in [17] for instance. Indeed, the strain field is expected to be rigorously equal to zero in this case. Figure 2-a shows an example for which parasitic fringes are clearly visible. This quasi-periodic noise is thought to be due to some signal sampling issues. The scale is adjusted in order to clearly see these fringes, but it is worth noting that their actual amplitude is small compared to the detection threshold of the 
technique employed here to measure strain. They could however constitute a concern in cases for which actual details in strain maps have the same order of magnitude as the amplitude of this quasi-periodic noise. Removing this quasi-periodic noise from the strain map can be carried out by applying the procedure described above. The spectrum is first calculated (see Figure 2-b). Even though a log scale has been used to reduce the difference in amplitude between these coefficients, only some bright spots located close to the center of this figure are visible. It means that the norm of the coefficients is highly decreasing when deviating from the center, and this is typically the case for many pictures. A close-up view of this zone is shown in Figure 3-a. Two peaks of Fourier coefficients clearly appear in this map near the center, along the direction of the fringe propagation. They correspond to the fringes, the remaining values being the noise due to other causes, namely sensor noise, quantization noise and slight grid defects. Since the fringes do not exactly describe a sine function, we rather have two clusters of peaks which are close and symmetric with respect to the center. Considering the uppermost top cluster, the distance $d$ between the highest peak along the $y$-direction and the center is equal to 9 pixels. Dividing by the height of the images (1401 pixels) provides an estimation of the frequency of the fringes: $9 / 1401 \simeq 6.4210^{-3}$ pixels $^{-1}$, and thus of the period: $1401 / 9 \simeq 156$ pixels. This last result can be easily checked in Figure 2-a, by measuring the average distance between two consecutive fringes along the $y$-direction.

It has been observed that all Procedures 1-5 described above lead to very similar results for this example. The reason is certainly that reducing significantly the few coefficients actually driving the quasi-periodic pattern of the undesirable fringes is enough here, the influence of the surrounding coefficients having only a very limited influence on the actual strain field, which is nearly null. Only results obtained with Procedures 2a, 4 and 5 are given here as examples of this filtering technique. For Procedure 5 , we have $\sigma_{u}=\sigma_{v}=3, \theta=0$, the zone to be corrected being a $9 \times 9$ square.

Figures $3-\mathrm{b}, \mathrm{c}$ and $-\mathrm{d}$ show a close-up of the spectrum in the same zone as in Figure 3-a. The effect of the correction is clearly visible. In case of Procedure-2a, (see Figure 3-b), all coefficients are identical within the zone, so the color is uniform within the zone. It is worth noting that the color is dark blue, thus meaning that the norm is very small, while the mean color around the zone (where the mean value is calculated) is brighter. The reason is that the norm is represented in the amplitude spectrum, and the norm of the mean value is lower than or equal to the mean value of the norm. It is here significantly lower. With Procedure 4, we have a distribution which is consistent with the value around the zone, which illustrates the fact that the correction relies on the values for the coefficients along the border through an interpolation. With Procedure 5, it can be seen that the most severe correction is performed at the center of the zone, which is precisely the location of the apices of the peaks. The common feature of all these cases is that the highest peaks have been removed.

The effects of these three corrections on the initial map is shown in Figure 4-a, -b and -c. As a general remark, the filtered maps which are obtained are very similar. The correction is slightly less efficient close to the borders, especially with the last procedure (see for instance near the bottom left border of Figure 4-c), but the fringes have disappeared elsewhere. The resulting distributions enable us to see the resulting strain maps, which are expected to be null here since we deal with a mere translation test. Slight horizontal fringes are visible (amplitude less that $10^{-4}$ ) in addition to the random noise due to the camera sensor noise, which propagates through the displacement and strain calculation program. These slight fringes are due to very small grid defects that are not completely eliminated by the procedure described in [2]. Noise is spatially correlated by the phase extraction procedure, as predicted and quantified in [18]. Some slight edge effects are also visible, since small residuals of the fringes still remain near the edges. Figure 5 finally summarizes the effect of this filtering procedure. The main result is that it is possible to split, within certain limits (edge effects in the current case), the initial map into two subimages: the first one contains the desired information and the second one the parasitic fringes.

\subsection{Example 2}

We consider here as a second example the strain map shown in Figure 6-a. This is a typical increment of longitudinal strain distribution between two consecutive images shot during a tensile test (dividing this map by the time step between two strain maps would provide an estimate of the strain rate). The tested specimen is made of aluminum alloy for which Portevin-Le Chatelier bands 
develop during plastic deformation. Isolated bands of straining fronts can be clearly observed, as well as fringes corrupting this map on the right-hand side. These fringes are assumed not to be caused by any physical reason, so they are considered as quasi-periodic noise which must be removed. The Fourier amplitude spectrum is depicted in Figure 7-a. Only a close-up view around the center of this distribution is shown, coefficients of higher harmonics being much lower in amplitude. Two clusters of peaks are clearly visible. They are symmetric with respect to the center and form a direction perpendicular to the mean direction of the fringes to be erased. As for the previous case, it can be checked that the main frequency associated with the clusters of peaks is consistent with the mean fringes period observed on the strain map. Procedures 1, 2, 3 and 5 described above give similar results (as long as parameters $\sigma_{u}, \sigma_{v}$ and $\theta=0$ are correctly adjusted for the last procedure), while Procedure 4 may lead to different results, depending on the size of the zone to be corrected. This choice is somewhat arbitrary, so the objective here is to illustrate how the combination of the choice of this zone and the settings of the parameters which drive some procedure (typically $\sigma_{u}, \sigma_{v}$ and $\theta=0$ with Procedure 5) influence the corrected spectrum and thus the corrected map. Only Procedures $2 \mathrm{a}, 4$ and 5 are considered here for the sake of consistency.

Figure 7-b,-c, and -d show a close-up of the distribution of the coefficients near the center after correction using Procedures 2, 4 and 5, a "small" zone $\left(38 \times 13\right.$ pixels $\left.^{2}\right)$ only being considered for the correction. The parameters driving the shape of the Gaussian notch filter are: $\sigma_{u}=45, \sigma_{v}=15$ and $\theta=-\frac{\pi}{12}$. The impact of the choice of the strategy chosen for correcting the spectrum is clearly visible by comparing in turn the corrected spectrums with the initial one. Remarks similar to those made in the first example can be drawn here: the correction is somewhat abrupt with Procedure $2 \mathrm{a}$ whereas a much smoother evolution of the norm of the coefficients can be observed with Procedures 4 and 5. It can also be seen that only the center of the cluster is affected by the corrections. This is due to the small dimension of the selected zone.

The effect of the corrections with these three procedures can be seen by comparing the filtered strain maps in Figures 6-b, c- and -d with the initial strain map in Figure 6-a. First of all, all these corrected maps are similar. However, some slight parasitic waves are visible with Procedure 4 (see Figure 6-c). They are likely to be caused by the "diffusion" inside the zone of significant Fourier coefficients localized around the zone, as observed in Figure 7-c.

We consider now a bigger zone, especially in terms of height: $45 \times 30$ pixels $^{2}$. The increase in area between these two strategies can be observed by comparing Figures 7-b,-c, and -d and Figures 8-b,-c, and -d, respectively. With Procedure 4, it is worth noting that the coefficients lying in the corrected zone are influenced by some coefficients located at the boundary which feature a significant norm: bidirectional interpolation leads to a "propagation" of these coefficients, which appear within the zone, along two inclined portions of straight lines (see Figure 8-c). The effect is visible in Figure 9-c, with slight parasitic waves (or "folds") propagating along a direction perpendicular to these straight lines. On the contrary, the Gaussian notch filter covers a bigger zone, so the coefficients featuring a significant norm are now more efficiently erased. Comparing Figures 6-c and 9-c enables us to assess the impact of the size of the zone. In particular, the "orange peel" appearance has disappeared, as a logical consequence of the fact that coefficients located further away from the center of the spectrum are affected by the biggest zone. Note finally that Figures 9-c and 9-d are similar, but some very slight waves parallel to and on the left-hand side of the strain band are more visible in the former case, which seems to indicate that Procedure 5 is slightly more efficient than Procedure 2 in this case. Figure 10 finally summarizes the effect of this filtering procedure involving the Fourier domain, thus avoiding the "classic" local spatial averaging which would have blurred the local details in the strain map.

\subsection{Example 3}

We consider as a last example the strain field occurring near the tip of a narrow vertical notch milled at the center of the soffit of a beam subjected to three-point bending. The notch tends to open and this leads strain concentration to occur near the tip. Figure 11-a thus shows the vertical strain field $\epsilon_{y y}$. Here, parasitic fringes are also superimposed to the actual strain distribution. They are due to severe grid defects which are not completely erased by the procedure described in [2]. Figure 11-b shows the map obtained after applying Procedure 1 described above and Figure 11-c the fringes which have been removed. The fringes are in this example much more regular than in 
the preceding examples. One can therefore apply a vertical one-pixel width spatial average filter, whose span is the period of the fringes, namely 29 pixels in the current case. This leads to the map shown in Figure 11-d. This map is smoother than the one shown in Figure 11-b. The reason is that high frequencies are canceled out with the spatial average filter which behaves like a low-pass filter. The caveat is that actual details which feature a characteristic length comparable to or lower than the span of the filter to be affected at the same time, thus leading to underestimate the strain amplitude. For instance, the strain value at the strain peak (point A) is equal to $-1.05 \%$ in Figure 11-b and -0.97\% in Figure 11-d. The relative difference is small (8\%), but underestimating strain may lead to underestimate stress at this critical point and this may be potentially an issue.

\section{Conclusion}

This paper recalls the basics of the discrete 2D Fourier transform and its application to remove parasitic fringes that often corrupt strain maps obtained with full-field measuring techniques. Various procedures have been tested. All of them consist in reducing (even zeroing) the norm of the Fourier coefficients corresponding to these fringes. These procedures differ by the route which is employed to change these values. Even though some limitations appear in some cases, it is shown through the examples shown in this paper that this approach is very easy to implement and leads to efficiently remove parasitic fringes in strain maps.

\section{Acknowledgements}

Dr. S. Amziane, Dr. E. Toussaint and Mrs I. Ben Cheikh are gratefully acknowledged for there help in obtaining some of the strain maps shown in this paper. 


\section{Annex: Matlab implementation}

The Matlab package has several native functions that turn out to be very useful to calculate the discrete Fourier transform of images, process them over certain zones and calculate the inverse Fourier transform of spectra. These functions are listed below. The reader is invited to refer to $[19,20]$ for further information.

- $\mathrm{fft} 2(\mathrm{~A})$ provides the $2 \mathrm{D}$ discrete Fourier transform of any matrix $\mathrm{A}$, which is a strain map in the current context

- $\mathrm{fftshift}(\mathrm{B})$ shifts any matrix B in such a way that its origin is at the center of the figure. B is a spectrum in the current context

- ifftshift(B) is the inverse of the previous function

- ifft2(B) provides the inverse Fourier transform of any matrix B, which is a spectrum in the current context

- griddata fits data known at a series of points and interpolates the obtained surface at given points. In the current context, this function is used in Procedure 4 to bilinearly interpolate over the zone to be filtered in the spectrum the Fourier coefficient located around this zone 


\section{References}

[1] M. Grédiac and F. Hild, editors. Full-field measurements and identification in solid mechanics. Wiley, novembre 2012. ISBN: 9781848212947, 496 pages.

[2] C. Badulescu, M. Grédiac, and J.-D. Mathias. Investigation of the grid method for accurate in-plane strain measurement. Measurement Science and Technology, 20(9):095102, 2009.

[3] I. Aizenberg and C. Butakoff. A windowed Gaussian notch filter for quasi-periodic noise removal. Image and Vision Computing, 26(10):1347-1353, 2008.

[4] M.A. Sutton, J.-J. Orteu, and H.W. Schreier. Image Correlation for Shape, Motion and Deformation Measurements - Basic Concepts, Theory and Applications. Springer, 2009.

[5] J. Dautriat, M. Bornert, N. Gland, A. Dimanov, and J. Raphanel. Localized deformation induced by heterogeneities in porous carbonate analysed by multi-scale digital image correlation. Tectonophysics, 503:100-116, 2011.

[6] S. Avril, P. Feissel, F. Pierron, and P. Villon. Estimation of the strain field from full-field displacement noisy data. European Journal of Computational Mechanics, 17(5-7):857-868, 2008.

[7] M. Lebrun, M. Colom, A. Buades, and J.-M. Morel. Secrets of image denoising cuisine. Acta Numerica, 21:475-576, 52012.

[8] P. Milanfar. A tour of modern image filtering: New insights and methods, both practical and theoretical. IEEE Signal Processing Magazine, 30(1):106-128, 2013.

[9] J. Fehrenbach, P. Weiss, and C. Lorenzo. Variational algorithms to remove stationary noise: Applications to microscopy imaging. Image Processing, IEEE Transactions on Image Processing, 21(10):4420-4430, 2012.

[10] F. Sur and M. Grédiac. Automated removal of quasiperiodic noise through frequency domain statistics Journal of Electronic Imaging, 24(1):013003, 2015. doi: 10.1117/1.JEI.24.1.013003. Society of Photo-Optical Instrumentation Engineers.

[11] M. Grédiac, E. Toussaint, C. Petit, A. Millien, and D. C. Nguyen. A comparative study of the heterogeneous local mechanical response of two types of asphalt mixes. Materials and Structures, 47(9):1513-1529,2014.

[12] A. van der Schaaf and J. van Hateren. Modelling the power spectra of natural images: Statistics and information. Vision Research, 36(17):2759-2770, 1996.

[13] R.C. Gonzalez and R.E. Woods. Digital Image Processing (3rd edition). Prentice Hall, Upper Saddle River, NJ, 2008.

[14] S. Kaur and R. Singh. An efficient method for periodic vertical banding noise removal in satellite images. International Journal on Computer Science and Engineering, 4(10):1710$1721,2012$.

[15] J. Wang and D.C. Liu. 2D FFT periodic noise removal on strain images. Proceedings of the 4th International Conference on Bioinformatics and Biomedical Engineering (ICBBE), Chengdu, China, pp 1-4, 2010.

[16] G.A. Al Hudhud and M. Turner. Digital removal of power frequency artifacts using a Fourier space median filter. IEEE Signal Processing Letters, 12(8):573-576, 2005.

[17] M. Grédiac and F. Sur. Effect of sensor noise on the resolution and spatial resolution of the displacement and strain maps obtained with the grid method. Strain, 50(1):1-27, 2014. Paper invited for the 50th anniversary of the journal. Wiley. 
[18] F. Sur and M. Grédiac. Towards deconvolution to enhance the grid method for in-plane strain measurement. Inverse Problems and Imaging, 8(1):259-291, 2014. American Institute of Mathematical Sciences.

[19] MATLAB. version 8.3 (R2014a). The MathWorks Inc., 2014.

[20] R.C. Gonzalez, R.E. Woods, and S.L. Eddins. Digital Image Processing Using MATLAB (2nd edition). Gatesmark Publishing, Knoxville, TN, 2009. 


\section{List of Figures}

1 Example of strain map [11] and corresponding amplitude spectrum and phase dis-

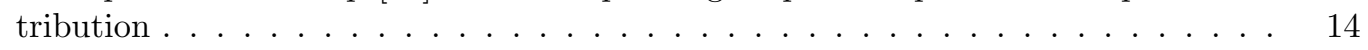

2 Example 1. Fringes to be removed . . . . . . . . . . . . . . .

3 Example 1. Close-up view around the center of Figure 2-b. a- Initial spectrum, bcorrected with Procedure 2a, c- with Procedure 4, d- with Procedure $5\left(\sigma_{u}=\sigma_{v}=\right.$

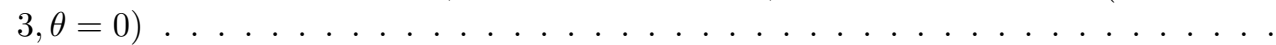

4 Example 1. Effect of the correction on the initial strain map. a- with Procedure 2a, c- with Procedure 4, d- with Procedure $5\left(\sigma_{u}=\sigma_{v}=3, \theta=0\right)$. . . . . . . . .

5 Example 1. Separation between sought information and parasitic fringes (Procedure $2 \mathrm{a}) \ldots \ldots \ldots \ldots \ldots \ldots \ldots$

6 Example 2. Effect of the corrections in Figure 7 on the initial strain map. ainitial strain map corrupted by parasitic fringes; b- strain map after filtering with Procedure 2a; c- strain map after filtering with Procedure 4, d- strain map after filtering with Procedure $5\left(\sigma_{u}=45, \sigma_{v}=15, \theta=-\frac{\pi}{12}\right) \ldots \ldots$

Example 2. Correction of a small zone of the spectrums. a- initial spectrum; bcorrected spectrum, Procedure 2a; c- corrected spectrum, Procedure 4, d- corrected spectrum, Procedure $5\left(\sigma_{u}=45, \sigma_{v}=15, \theta=-\frac{\pi}{12}\right) \ldots \ldots \ldots$

8 Example 2. Correction of a big zone of the spectrums. a- initial spectrum; bcorrected spectrum, Procedure 2a; c- corrected spectrum, Procedure 4, c- corrected spectrum, Procedure $5\left(\sigma_{u}=45, \sigma_{v}=15, \theta=-\frac{\pi}{12}\right) \ldots \ldots \ldots$

9 Example 2. Effect of the corrections in Figure 8 on the initial strain map. ainitial strain map corrupted by parasitic fringes; b- strain map after filtering with Procedure 2a; c- strain map after filtering with Procedure 4, d- strain map after filtering with Procedure $5\left(\sigma_{u}=45, \sigma_{v}=15, \theta=-\frac{\pi}{12}\right) \ldots \ldots \ldots$

10 Example 3. Separation between sought information and parasitic fringes (big zone,

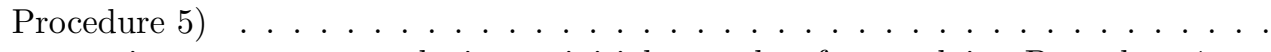

$11 \epsilon_{y y}$ strain map near a crack tip. a- initial map, b- after applying Procedure 1, c-: fringes which have been removed, d-: after applying a spatial average filter. . . . . 24 


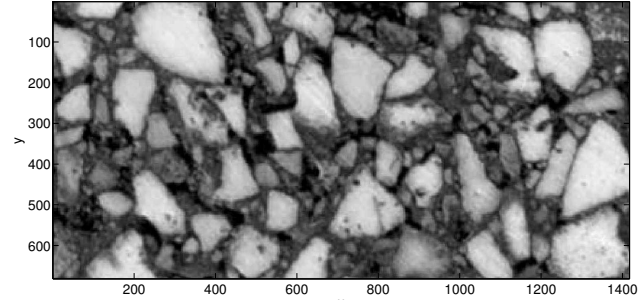

a- front face of the specimen (11)

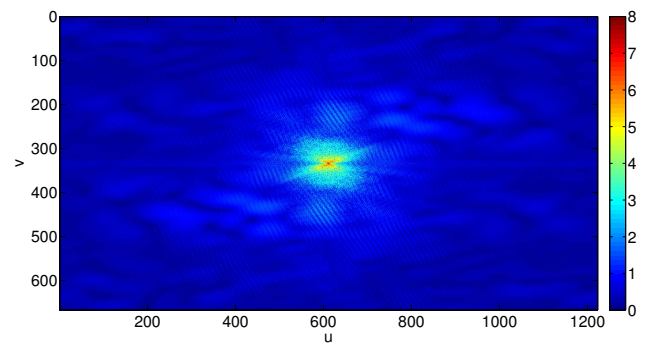

c- spectrum of the strain map

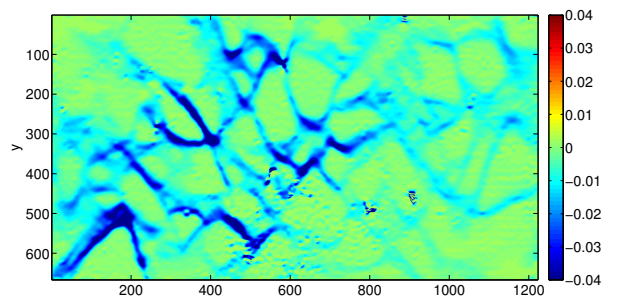

b- $\epsilon_{y y}$ strain map (11)

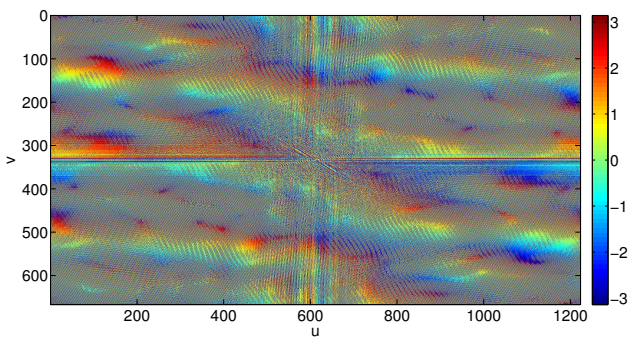

d- argument distribution

Figure 1: Example of strain map [11] and corresponding amplitude spectrum and phase distribution 


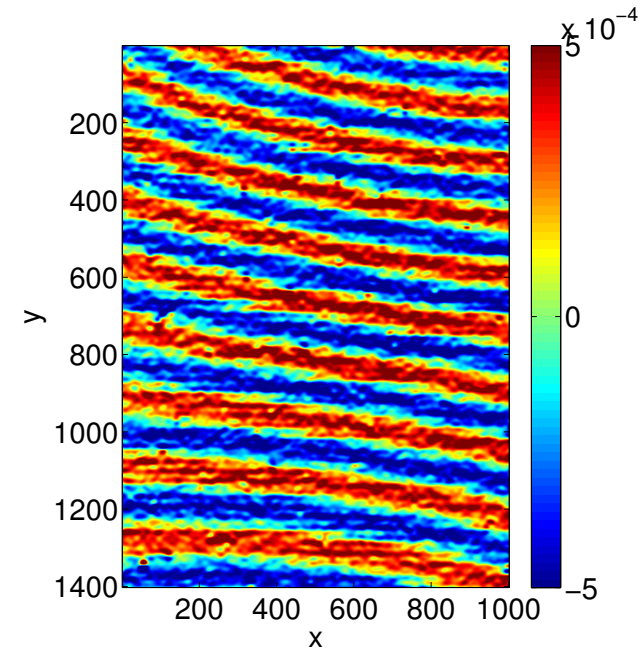

a- map to be filtered

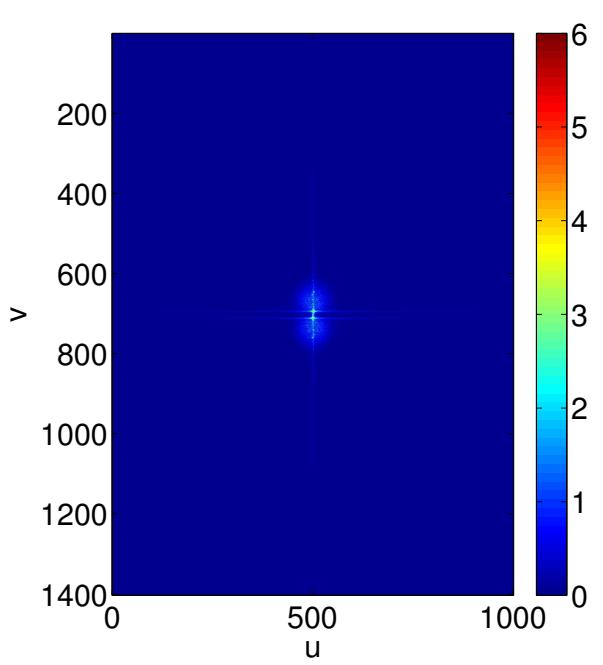

b- Fourier amplitude spectrum

Figure 2: Example 1. Fringes to be removed 


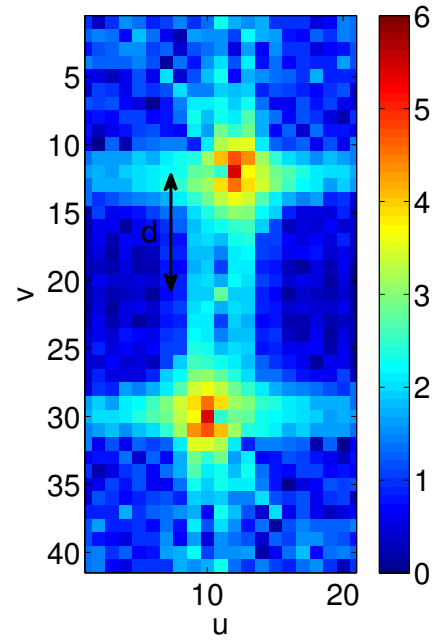

a-

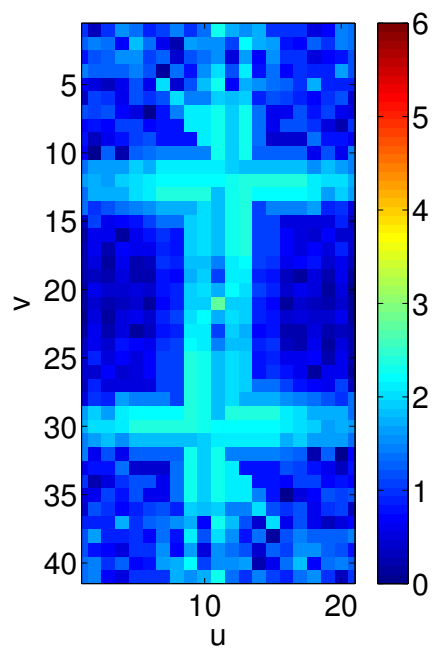

c-

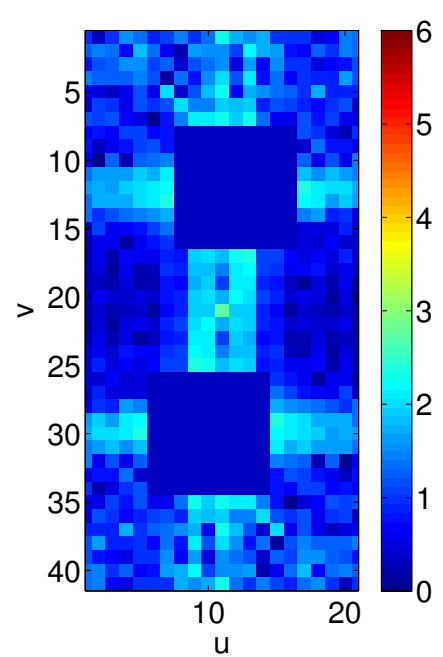

b-

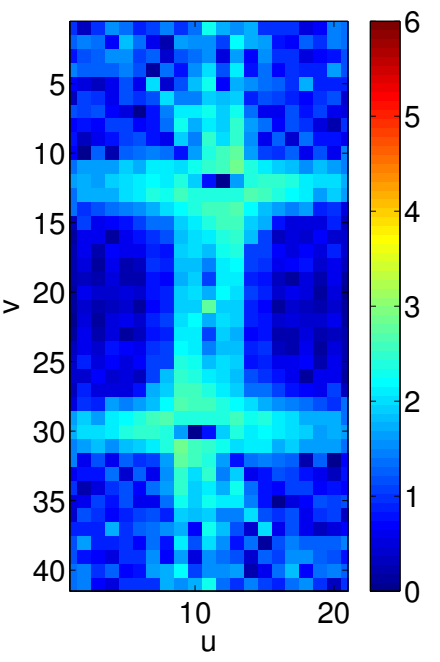

d-

Figure 3: Example 1. Close-up view around the center of Figure 2-b. a- Initial spectrum, bcorrected with Procedure 2a, c- with Procedure 4, d- with Procedure $5\left(\sigma_{u}=\sigma_{v}=3, \theta=0\right)$ 


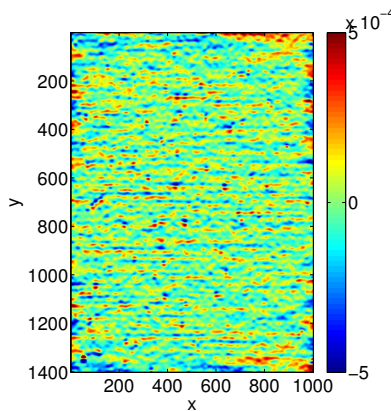

a-

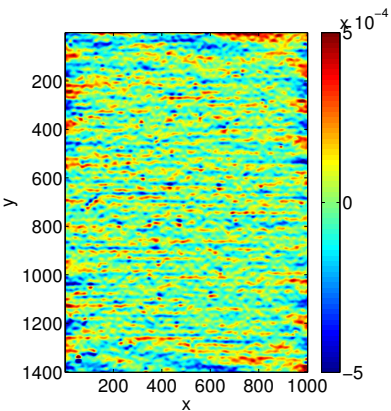

b-

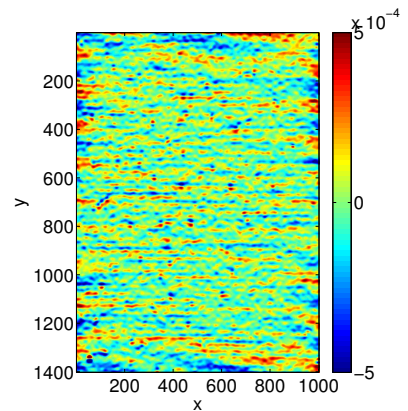

$c-$

Figure 4: Example 1. Effect of the correction on the initial strain map. a- with Procedure 2a, cwith Procedure 4 , d- with Procedure $5\left(\sigma_{u}=\sigma_{v}=3, \theta=0\right)$ 

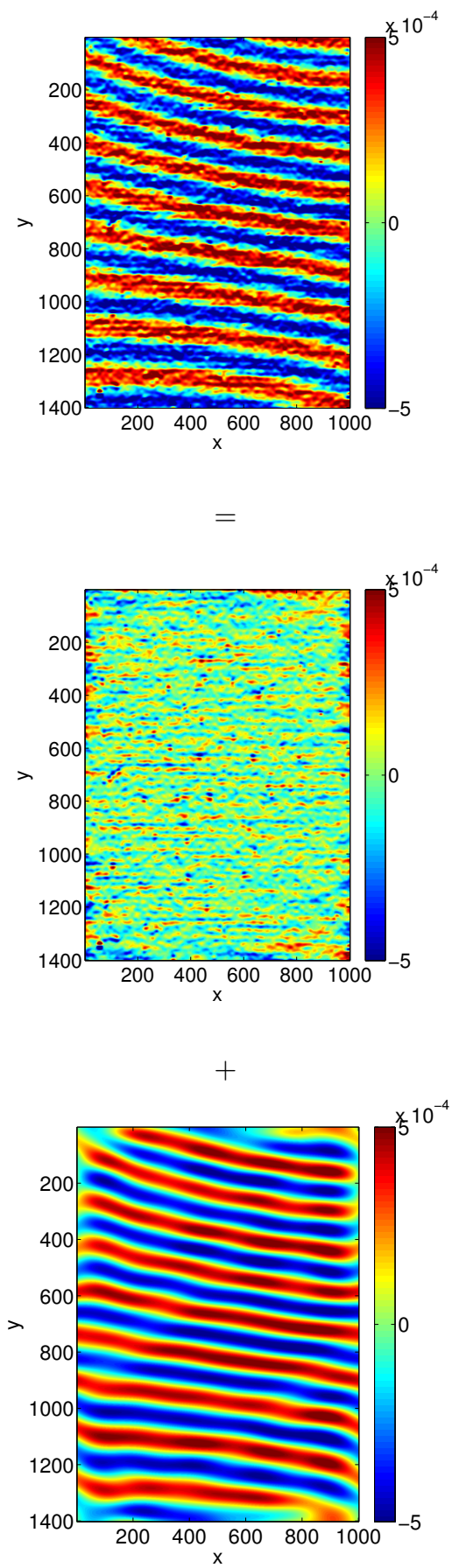

Figure 5: Example 1. Separation between sought information and parasitic fringes (Procedure 2a) 


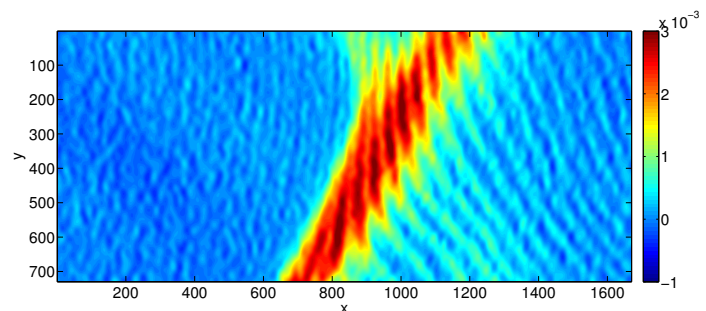

a-

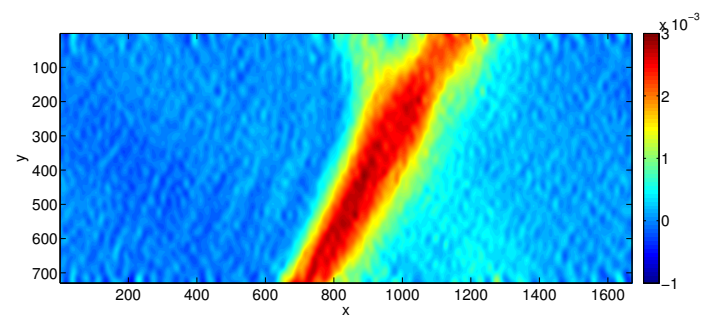

c-

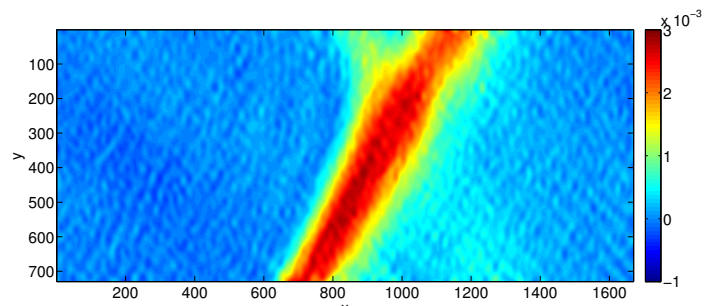

b-

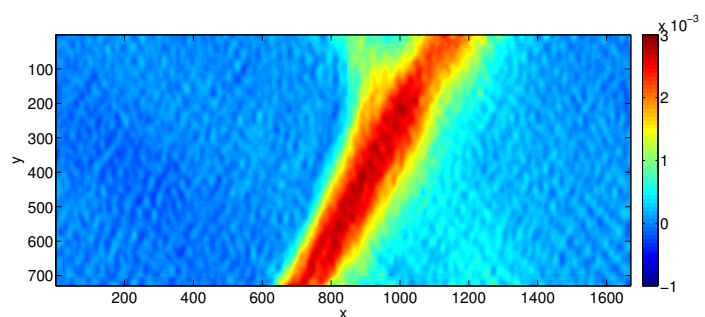

d-

Figure 6: Example 2. Effect of the corrections in Figure 7 on the initial strain map. a- initial strain map corrupted by parasitic fringes; b- strain map after filtering with Procedure 2a; c- strain map after filtering with Procedure 4, d- strain map after filtering with Procedure $5\left(\sigma_{u}=45, \sigma_{v}=15\right.$, $\left.\theta=-\frac{\pi}{12}\right)$ 


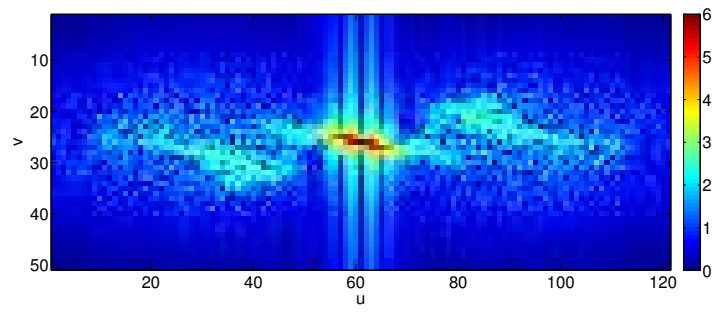

a-

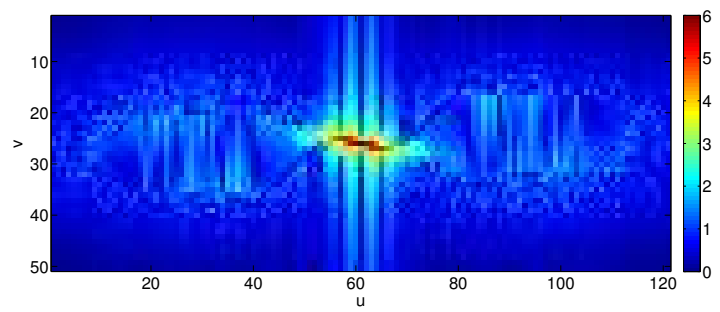

c-

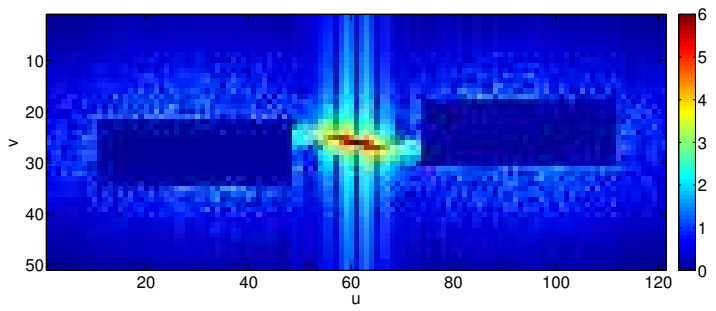

b-

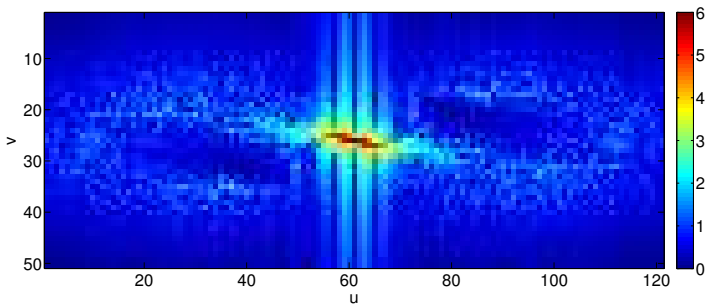

d-

Figure 7: Example 2. Correction of a small zone of the spectrums. a- initial spectrum; b- corrected spectrum, Procedure 2a; c- corrected spectrum, Procedure 4, d- corrected spectrum, Procedure 5 $\left(\sigma_{u}=45, \sigma_{v}=15, \theta=-\frac{\pi}{12}\right)$ 


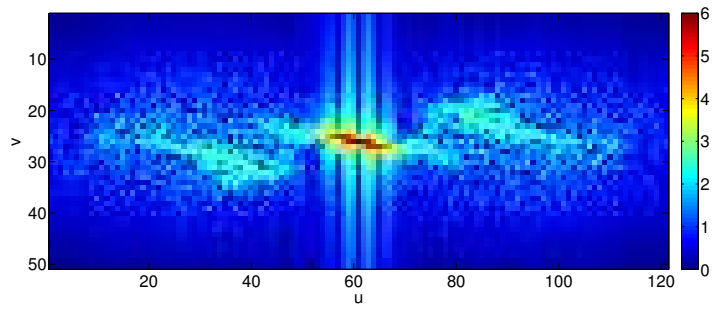

a-

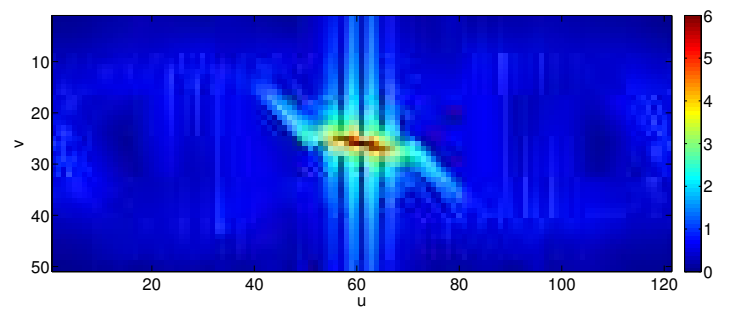

C-

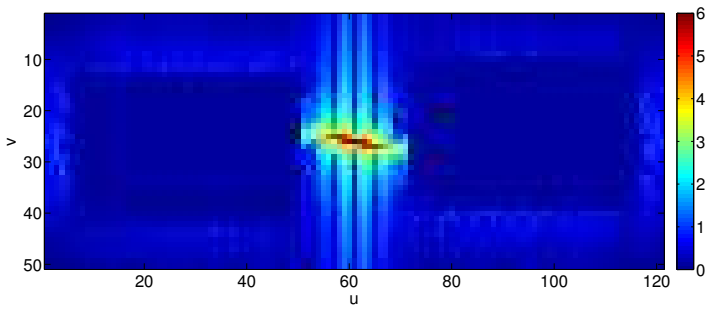

b-

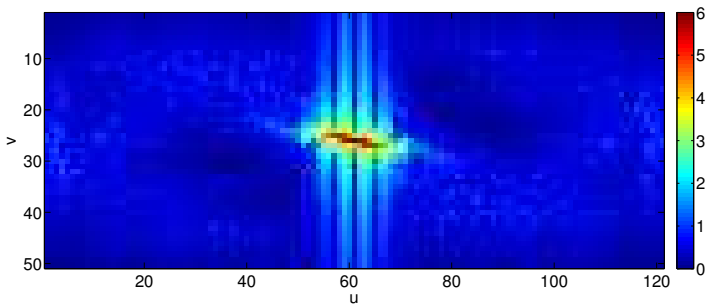

d-

Figure 8: Example 2. Correction of a big zone of the spectrums. a- initial spectrum; b- corrected spectrum, Procedure 2a; c- corrected spectrum, Procedure 4, c- corrected spectrum, Procedure 5 $\left(\sigma_{u}=45, \sigma_{v}=15, \theta=-\frac{\pi}{12}\right)$ 


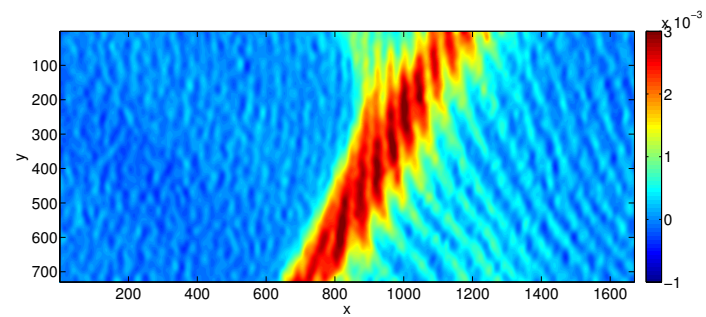

a-

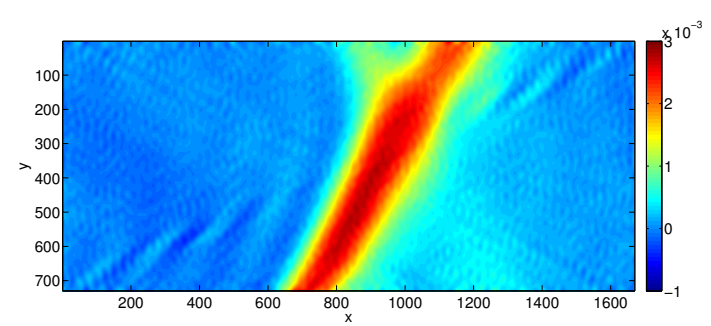

C-

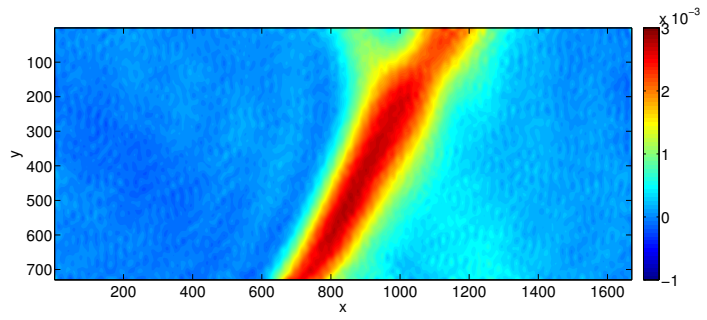

b-

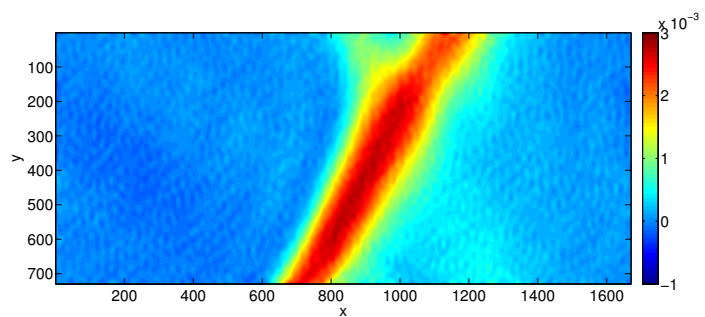

d-

Figure 9: Example 2. Effect of the corrections in Figure 8 on the initial strain map. a- initial strain map corrupted by parasitic fringes; b- strain map after filtering with Procedure 2a; c- strain map after filtering with Procedure 4, d- strain map after filtering with Procedure $5\left(\sigma_{u}=45, \sigma_{v}=15\right.$, $\left.\theta=-\frac{\pi}{12}\right)$ 


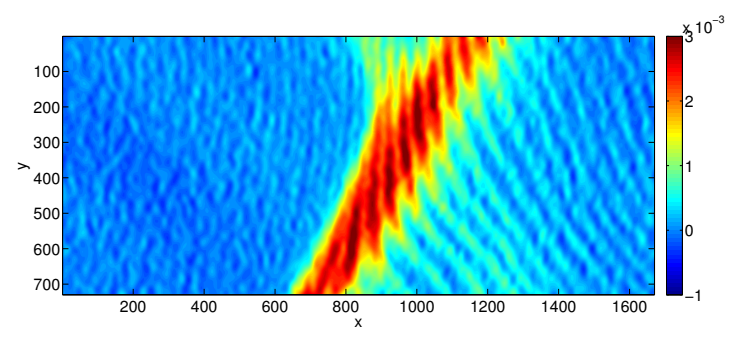

$=$

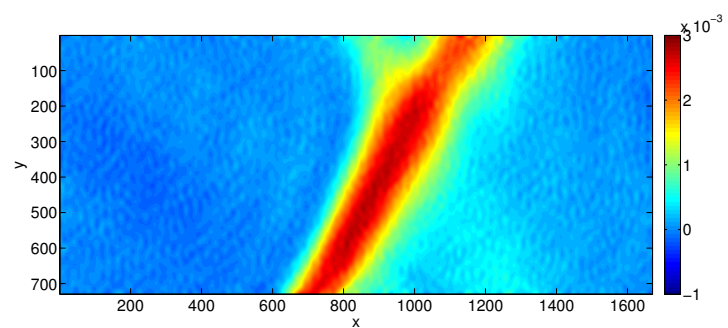

$+$

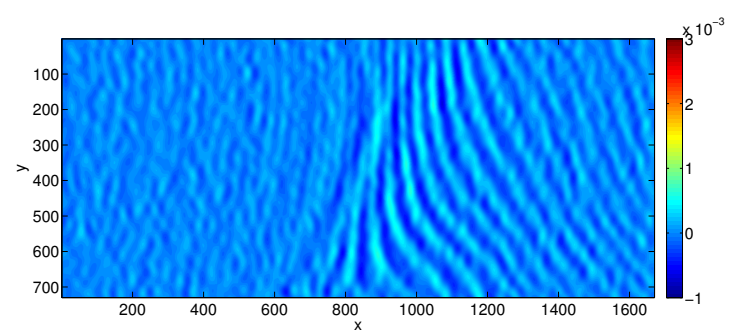

Figure 10: Example 3. Separation between sought information and parasitic fringes (big zone, Procedure 5) 


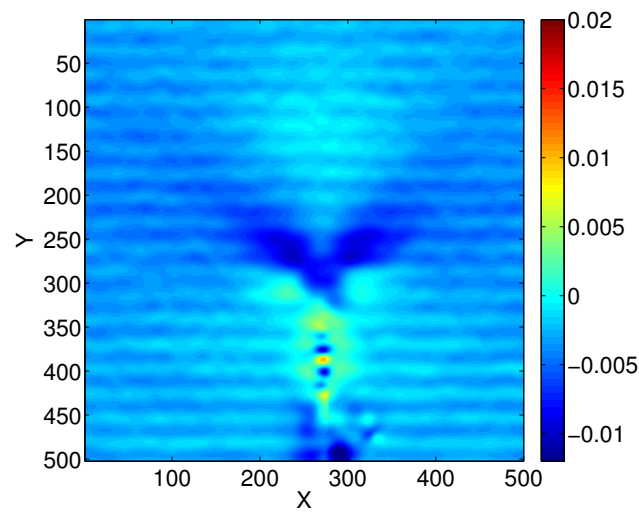

a-

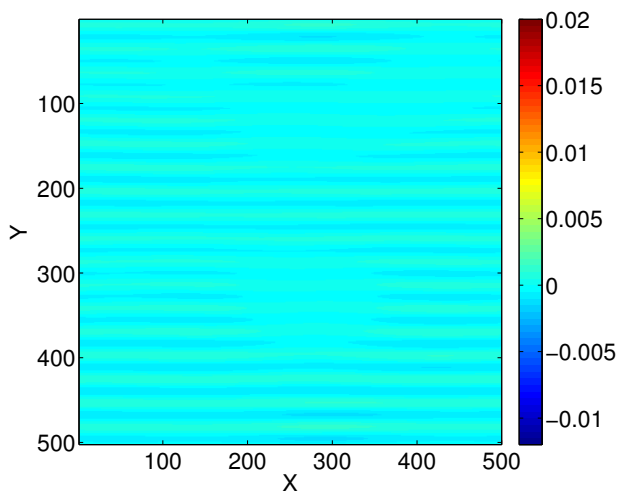

c-

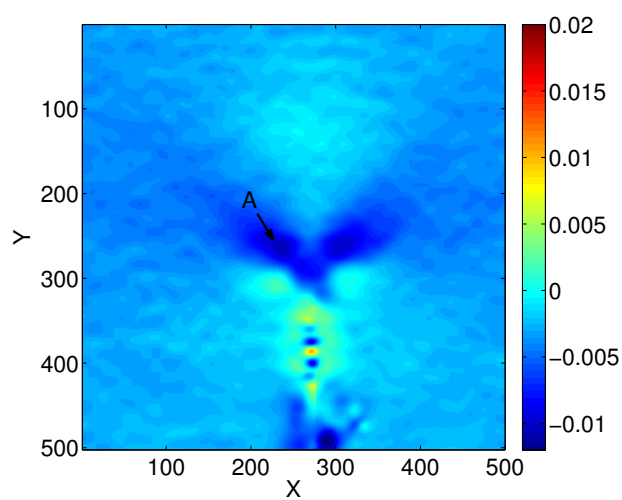

b-

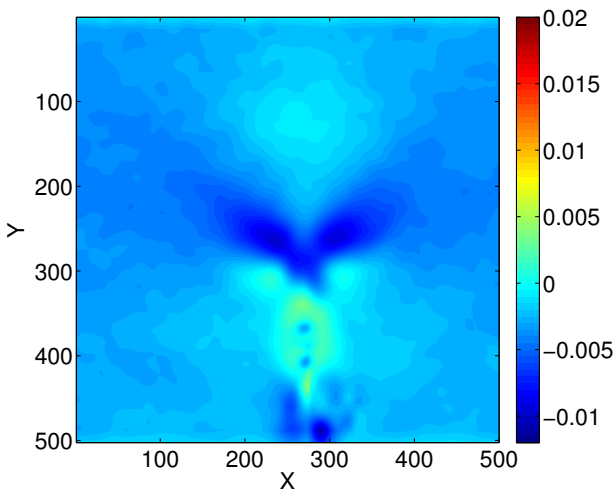

d-

Figure 11: $\epsilon_{y y}$ strain map near a crack tip. a- initial map, b- after applying Procedure 1, c-: fringes which have been removed, d-: after applying a spatial average filter. 